\title{
Moral Distress and Related Factors Among Emergency Department Nurses
}

\author{
(1) Kamyar Jalali, (1) Rasoul Tabari-Khomeiran, (1) Fariba Asgari, (1) Mitra Sedghi-Sabet, (1) Ehsan Kazemnejad
}

Social Determinants of Health Research Center, Guilan University of Medical Sciences, Rasht, Iran

\begin{abstract}
Aim: The study determines moral distress and related factors among emergency nurses. Moral distress is one of the recurring issues in the nursing profession that has gained importance by creating changes in the health care system.

Materials and Methods: An analytic-descriptive study was conducted, in which all 180 nurses working in emergency education and health centers in were included. Moral distress was measured by questionnaire. Data were analyzed using SPSS 17.

Results: The findings showed that moral distress in emergency nurses (4.93 \pm 1.08$)$ was high. Most moral distress was in the area of professional-functional competence $(5.21 \pm 1.17)$ in the item of "unsafe conditions" (5.55 \pm 1.45$)$. No statistically significant correlation was found between age and experience with moral distress among nurses in the emergency department. From the perspective of nurses in the study, organizational factors including barriers to education were the strongest factor influencing moral distress.
\end{abstract}

Conclusion: Given that moral distress in nurses participating in the study was very high, it seems that planning to promote professionalfunctional competency of nursing practice are essential.

Keywords: Moral distress, nursing ethics, emergency department services, emergency de details

\section{Introduction}

Any profession that is linked directly with clients' needs morality; although ethics is essential in all jobs, it is an inseparable part of the nursing profession, because moral behavior and responsibility in nurses with patients has a significant role in improving their health (1-3).

In the nursing profession, because of long communication with the client and direct responsibility for patients, nurses are faced daily with ethical decisions that usually raise the following questions: Have you ever decided to do the best thing for the patient but felt that you cannot do it? Have you ever wanted not to implement the law because of policies and procedures that prevent the best thing for the patient? If you have had any of these feelings, you have experienced moral distress $(1,3)$.
Moral distress was first described by Jameton as when the nurse knows what the right action is, but organizational politics and conflict with colleagues prevent him/her from doing the right thing. In a study by de Veer et al., (2) moral distress was distinguished from emotional distress. A nurse may experience emotional distress while restrict the patient, but if the nurse believes that restriction of patient is not morally correct then he/she experiences moral distress. Moral distress is a concern for nurses around the world (4). Statistics indicate that one out of every three nurses experiences moral distress. Additionally, at least one out of ten people will leave the profession because of moral distress. A considerable number of nurses face ethical challenges in their everyday practice $(5,6)$.

Moral distress can derive from a variety of sources, including limited resources, organizational policies, direct communication with patients, providing unnecessary medical care, hospital 
procedures, more social demand, an affront to the independence of patients, relations between doctors and nurses, conflicts with the wishes of the family, decisions on the release of patient's information, and the inability to prevent the patient's death $(7,8)$.

In this regard, Kazemi et al. (9) study indicated that the existence of conflicts and differences of opinion between medical staff and patients' relatives about treatments that are useless or less useful in the medical staff's opinion, but which the patient or those around him want to apply, and on the other hand, medical advice and treatments that the medical staff know to be useful but which the patient or his relatives would not accept as medical expenses, cause moral distress to manifest in nurses.

As a result of these factors, nurses experiencing moral distress react in different ways. Some go along with other treatment team members and avoid unnecessary treatments, while others remain silent. Some will experience physical and psychological symptoms. Physical symptoms due to moral distress have been reported as palpitations, headaches, sleep disturbances, and changes in bodily functions. Psychological consequences are seen in the form of feelings of imbalance, low self-esteem, guilt, regret, anxiety, hopelessness, lack of energy, emotional exhaustion, withdrawal from family and friends, and deep sorrow $(10,11)$.

Corel's theory proposes a model of moral distress in which, if a person has the moral courage to do the right thing, he/she will experience moral discomfort. Moral distress has different effects on nurses, patients, and health systems, and its consequences are important: in nurses, it causes them to leave the profession, and to experience isolation and burnout; when patients are faced with loss of quality of care and lack of patient protection, their irritation and discomfort will increase. Finally, organizations also suffer from the effects of this problem and are faced with heavy costs because of the loss of staff and difficulty recruiting new staff, which leads to decreases in quality of care and lack of patient satisfaction, which can endanger their professional reputation $(12,13)$. The results of Fernandez-Parsons et al.' (14) study at emergency sectors of hospitals in California, America demonstrated low intensity of moral distress (14), while Ohnishi et al. (15) study in Japan's public hospitals showed high levels of moral distress. In the nursing community of [Country name], like other communities, moral distress is alarmingly high, and among the special sectors, emergency and ICU have a high degree of distress (16). In these sectors, moral distress in nurses occurs because of the special conditions of patients and the emergency status of medical operations (Diagnosis-Treatment), and nurses are exposed to moral distress more than in other sectors. Studies suggest that nurses experience different levels of moral distress (17). Beikmoradi et al. (18) in a cross sectional study conducted on
163 nurses working in intensive care units and medical training centers, reported a high level of moral distress. Ebrahimi and colleagues, in a descriptive-analytic study on 418 nurses working in teaching and medical hospitals, reported a moderate to high level of moral distress, and concluded that among personal features, job status and educational level were significantly associated with moral distress (19). Ameri et al. (5) reported high levels of moral distress and reported significant correlations between nurses' age, experience, and type of employment, and the intensity of moral distress.

The study of moral distress in emergency nurses is important, because the emergency room is where transfer of ill patients from pre-hospital emergency medical centers occurs, while on the other hand, nurses bear the task of stabilizing the vital signs of patients entering the clinical, special, and operation departments of the hospital and other hospitals. In addition, the sector is faced with a host of outpatients who for various reasons, have chosen emergency treatment and who expect to receive timely and high-quality services $(20,21)$. Although in recent years, moral distress experienced by nurses in different parts has received attention, no study on this issue in relation to moral distress has been conducted in. Therefore, this study has been designed and implemented to investigate moral distress and related factors in the emergency departments and medical educational centers in.

\section{Materials and Methods}

This is a cross-sectional study (with a descriptive-analytic approach) that was conducted with 192 nurses working in emergency departments and training centers in. Hundred and eighty nurses were enrolled after providing informed consent via the census method. A total of 12 participants were excluded because of the exclusion criteria, no-filling, sickness, and maternity leave. The researcher referred to units in the morning, afternoon and evening shifts and data were collected at the beginning, about work purposes, confidential boxes, inclusion and exclusion criteria (experience of less than one year, maternity leave, sickness more than a month, and students), and then by identifying target nursing staff, explanation about the purpose of the work (defined subject and scoring), and data safe boxes (reason: the secrecy and lack of accountability of colleagues in questionnaires), and if these people agreed to complete the informed consent form.

Data collection tools included a two-part questionnaire, which consisted of a standardized questionnaire to measure moral distress scale and its related factors. The scale showed acceptable validity and reliability according to Razzaghi Kashani et al. (16). 
The related factors questionnaire comprised three areas of demographic, occupational-social, and organizational factors, and included 18 questions about the subjects (age, gender, marital status, level of education, experience, position, income, employment status, interaction between physicians and nurses, the interaction between nurses, supporting one's parents and wife or not, hospital management support, job promotion opportunities, Job security, arrangements for continuing education, further education barriers, and stress and tension).

The Jamitoon moral distress scale is the first scale assessing moral distress in the community of nurses. This questionnaire consists of 30 questions in three areas, including ignoring the patient (Cronbach's alpha of 0.92) with 16 questions, patient's decision-making power (Cronbach's alpha 0.86) with 8 questions, and professional and functional competence (Cronbach's alpha of 0.79) with 6 questions. The scale measures the amount of moral distress that nurses experience in specific locations and conditions. The scoring of this tool uses a 7-point Likert format ranging from very low levels of moral distress to very high. In the questionnaire, it was stated that, if a respondent had not had any experience of a specific ethical problem, they should leave that item blank, and if they had faced an ethical problem, to rate it on a scale ranging from the lowest (1) to the highest (7). The range of the score of each area is from 1 to 7 , and the total score of the questionnaire is also between 1 and 7 ; a total average score closer to 7 indicates higher moral distress, while a total average score closer to 1 indicates lower moral distress. The response variable used in this study was that a score less than the average was considered to indicate low moral distress and a score above average was considered to indicate high moral distress $(m=4.93)$. The scientific validity and reliability of the moral distress measurement tool was measured by Dr. Razzaghi Kashani et al. (16) in 2008, who determined this using Cronbach's alpha of $86 \%$ and a re-test.

Guilan University of Medical Sciences Institutional Review Board approved this study, in accordance to Helsinki Declaration (approval number: IRBGUMS29308264).

\section{Statistical Analysis}

Data were analyzed using SPSS 17 software; descriptive statistics (percent) and means (standard deviation) were calculated, and the relationships between specific factors and moral distress ratings were examined using inferential statistical tests (t-test and ANOVA) considering $p<0.05$.

\section{Results}

In this study, 180 of 192 nurses completed the questionnaires; 93.9\% (169 people) of respondents were female, and 5\% (9 people)
Table 1. Distribution of demographic and occupational - social characteristics of participants

\begin{tabular}{|c|c|c|c|}
\hline Characteristics $(n=180)$ & & $\mathbf{n}$ & $\%$ \\
\hline \multirow{3}{*}{ Age (years) } & $22-28$ & 78 & 43.3 \\
\hline & $29-35$ & 54 & 30 \\
\hline & $>35$ & 48 & 26.7 \\
\hline \multirow{2}{*}{ Marital status } & Single & 80 & 44.4 \\
\hline & Married & 96 & 53.3 \\
\hline \multirow{3}{*}{ Educational status } & Associate degree & 2 & 1.1 \\
\hline & Bachelor & 174 & 96.7 \\
\hline & Master & 4 & 2.2 \\
\hline \multirow{4}{*}{ Type of employment } & Employed & 48 & 26.7 \\
\hline & Long term contract & 43 & 23.9 \\
\hline & Short term contract & 38 & 20.6 \\
\hline & Formal obligation & 50 & 27.8 \\
\hline \multirow{3}{*}{ Work experience (years) } & $1-7$ & 95 & 52.8 \\
\hline & 8-14 & 37 & 20.5 \\
\hline & $>14$ & 48 & 26.7 \\
\hline \multirow{2}{*}{ Clinical position } & Head nurse & 9 & 5 \\
\hline & Clinical nurse & 170 & 94.4 \\
\hline
\end{tabular}

Table 2. Estimated regression coefficients of effective factors (organizational factors) and rating of moral distress according to logistic model of listening-reading method

\begin{tabular}{|c|c|c|c|c|c|c|}
\hline \multirow{2}{*}{ Variable } & \multirow{2}{*}{$\beta$} & \multirow{2}{*}{ SE } & \multirow{2}{*}{$p$ value } & \multirow{2}{*}{ Odds ratio } & \multicolumn{2}{|l|}{$\mathrm{Cl}$} \\
\hline & & & & & $\mathrm{LL}$ & UL \\
\hline $\begin{array}{l}\text { Education } \\
\text { obstacle }\end{array}$ & 0.367 & 0.171 & 0.032 & 1.443 & 1.032 & 2.018 \\
\hline
\end{tabular}

were male. The mean age and standard deviation of the subjects were $30.93 \pm 6.20$ and average and SD of work experience was $6.60 \pm 5.05$. Regarding marital status, $4.44 \%$ (80 cases) of nurses were single and 35.3\% (96 cases) were married. Thirty percent of respondents were employed in Poursina Hospital, 20\% in Razi, $1.16 \%$ in Heshmat, $7.8 \%$ in Velaayat, $1.1 \%$ in Alzahra, $6.7 \%$ in AmirAl-Momenin, $8.9 \%$ in Shafa, and 9.4\% in Hefdah-e-Shahrivar. In terms of education, $1.1 \%$ of those surveyed were technicians, 96.7\% were experts, and $2.2 \%$ had an MSc in nursing. In terms of employment status, $26.7 \%$ were in formal employment, $23.9 \%$ were in treaty employment, and $27.8 \%$ were employed on a contract basis for a plan. In terms of position, $5 \%$ were head nurses, $94.4 \%$ were nurses, and $0.6 \%$ gave no response. Among those surveyed in October or November, 3 people had maternity leave, 2 had exclusions, 4 people were employed less than 1 year, and 3 people were uncooperative.

According to the study, the average moral distress in nurses was $4.93 \pm 1.08$. Thus, high moral distress was reported. Heshmat hospital emergency department indicated the highest level of moral distress with a mean (standard deviation) of $5.20 \pm 1.30$ and emergency department of Hefdah-Shahrivar Hospital indicated 
the lowest with a mean (standard deviation) of $4.57 \pm 1.27$. Among the areas of moral distress, professional and functional competence was assigned the highest rating with an average of $5.21 \pm 1.17$. In this context, according to the nurses, the item "During care and treatment in unsafe conditions" with a rating of 5.55 and the item "Working in conditions in which, due to lack of nurses, insufficient care for the patient is done" with a rating of 5.49 showed the highest moral distress scores. For ignoring the patient, the item "providing better care for rich patients" with a rating of 4.28, while for patient's decision-making power, the item "avoids taking oral medication" with a rating of 4.37 showed the lowest moral distress scores in nurses.

Among the relevant factors, organizational factors such as barriers to further education $(p=0.04)$ and stress $(p=0.49)$ had significant relationships with nurses' moral distress. Nurses who applied effective stress combating strategies, had less distress than others. The regression coefficient of factors related to moral distress based on logistic regression model using imported Beck shows that among the factors related to moral distress with a significance level of $p=0.25$, only education barriers remained significant in the final model, so that by increasing education barriers, the chances of creating moral distress were almost 1.5 times higher. Additionally, personal factors with variables of age, gender, etc., and occupational-social factors with variables of scientific interaction between nurses, family support, etc., had no significant statistical relationships with intensity of moral distress.

The results showed that the education barriers variable $(p=0.032)$ significantly predicted nurses' moral distress. The direction of this impact is positive, and the results show that nurses' moral distress increases with increasing education barriers and the chance of creating moral distress in nurses increases almost 1.5 times (odds ratio $=1.443$ ).

\section{Discussion}

The results of this study show that nurses experience high average rates of moral distress. The results of this part of the study are comparable with other studies in this area. Abbaszadeh et al. (7) conducted a study in 2012 and reported high rates of moral distress (5.041) in nurses working in educational and health centers of. A study conducted in 2013 by Ameri et al. (22) reported high moral distress levels in nurses working in the oncology sector (2.13 of 4), which is consistent with the results of this study. However, a study by Fernandez-Parsons et al. (14) conducted in 2013 reported low levels of moral distress in the emergency department that does not match with the results of this study. This contradiction could be due to differences in the tools used, that is, differences in the type of wording and scoring method of each tool. Furthermore, nurses are faced daily with critical decisions about patients, and considering the definition of moral distress, which is an inner experience and its causes, relates to some inner individual features, and these characteristics are the basis of ones' willingness or unwillingness to solve ethical problems (23). Therefore, it seems that unique individual characteristics (personality, experience, and skills) of each sample may have caused contradictions in the moral distress for research units.

In the realm of moral distress, professional and functional competence of patients was associated with high moral distress. In this context, the items "Working in unsafe conditions because of lack of nurses" and "Working in a situation where due to the lack of nurses, there is inadequate care for the patients" had the highest ratings. The results of this study were consistent with the findings of Abbaszadeh et al (7) and co-workers about moral distress in nurses working in teaching hospitals in, while they were inconsistent with the study of Ameri et al. (22) and Allari et al. (24) concerning moral distress among nurses in Jordan and its relation to moral atmosphere. Perhaps considering the fact that half of the research units working in the emergency department are in a plan and contractual, it seems that employing nurses with low skills and experience caused the highest moral distress in nurses. Therefore, the lower the functional-professional competence, the higher the moral distress in nurses.

In this study, social-occupational and individual factors were not significantly associated with the intensity of moral distress. The results were different from those of Beikmoradi et al. (18) results that used MDS tools revised by Haamrik (18). This could be because of differences in study tools, and also because of differences in the environment investigated.

In logistic regression analysis, the only significant variable was education barriers, so it can be argued that education barriers are the most powerful predictor of moral distress in nurses, which means that increased education barriers cause nurses more moral distress. de Veer et al. (25) showed there are no predictor factors for moral distress in the daily performance in nurses. Evaluation with different tools may cause different results. Furthermore, given that there are more young people and novice nurses in the emergency department and they are interested in continuing their education, that they find education barriers stressful should be considered by corporate executives.

\section{Conclusion}

Intensity of moral distress in the emergency department nurses was evaluated to occur at a high level. Considering the outcome and impact of moral distress on nurses and hospital attendants 
and of subsequently dealing with it, creating areas of reduced moral distress should be considered for patients, special distress workshops for nurses should be held, and special courses taught to reduce moral distress in the nursing community. Given that moral distress was not significantly associated with individual factors, a plan of in-service distress measurement may be helpful. In the present study, nurses who must endure stress and tension in the workplace had high distress, which could indicate a lack of awareness of methods of controlling moral distress. Therefore, teaching strategies for dealing with moral distress can be very helpful. Given that the highest moral distress was expressed in the condition of shortages of nurses for patient care, planning to increase the number of nurses in hospital wards is required. The amount of moral distress in the professionalfunctional competence area is high. Therefore, planning to create professional-functional competency of nurses appears necessary. Considering that education barriers were one of the strong factors in creating moral distress, creating a learning environment in hospitals should be considered.

\section{Ethics}

Ethics Committee Approval: Guilan University of Medical Sciences, approval number: IRBGUMS29308264.

Informed Consent: It was taken.

Peer-review: Externally peer-reviewed.

\section{Authorship Contributions}

Surgical and Medical Practices: R.T.K., F.A., M.S.S., Concept: K.J., R.T.K., Design: R.T.K., E.K., Data Collection or Processing: K.J., E.K., Analysis or Interpretation: M.S.S., E.K., Literature Search: R.T.K., F.A., M.S.S., Writing: K.J., R.T.K., F.A.

Conflict of Interest: No conflict of interest was declared by the authors.

Financial Disclosure: The authors declared that this study was supported by the Guilan University of Medical Sciences [grant number: GUMS93080604].

\section{References}

1. Burston AS, Tuckett AG. Moral distress in nursing: contributing factors, outcomes and interventions. Nursing Ethics. 2013;20:312-24.

2. de Veer AJ, Francke AL, Struijs A, Willems DL. Determinants of moral distress in daily nursing practice: a cross sectional correlational questionnaire survey. Int J Nurs Stud. 2013;50:100-8.

3. Karagozoglu S, Yildirim G, Ozden D, Çınar Z. Moral distress in Turkish intensive care nurses. Nursing Ethics. 2017;24:209-24.

4. Ulrich $\mathrm{CM}$, Hamric $\mathrm{AB}$, Grady C. Moral distress: a growing problem in the health professions? The Hastings Cent Rep. 2010;40:20-2.
5. Ameri M, Safavi Bayat Z, Ashktorab T, Kavoosi A, Vaezi A. Moral distress: evaluating nurses' experiences. J Med Ethics Hist Med. 2013;6:64-73.

6. Ulrich CM, Taylor C, Soeken K, O’Donnell P, Farrar A, Danis M, et al. Everyday Ethics: Ethical Issues and Stress in Nursing Practice. J Adv Nurs. 2010;66:25109 .

7. Abbaszadeh AB, Borhani F, Kalantary S. The moral distress of nurses in health centers in the city of Bam in 1390. Iranian Journal of Medical Ethics. 2011;6.

8. Afshar MH , Jooybari L, Sanagou A, Kalantari S. Study Of Factors Affecting Moral Distress Among Nurses: A Review of Previous Studies. J Educ Ethics Nurs. 2012;1(1).

9. Kazemi AK, Kazemi M, Abbasi M, Kiyani M, Feyzollahi N, Atoloo S, et al. Assessing end of life support. Medical Ethics and History of Medicine. 2012;5:45-57.

10. Maluwa VM, Andre J, Ndebele P, Chilemba E. Moral distress in nursing practice in Malawi. Nurs Ethics. 2012;19:196-207.

11. Corley MC, Minick P, Elswick R, Jacobs M. Nurse moral distress and ethical work environment. Nursing Ethics. 2005;12:381-90.

12. Lamiani G, Borghi L, Argentero P. When healthcare professionals cannot do the right thing: A systematic review of moral distress and its correlates. J Health Psychol. 2017;22:51-67.

13. Morley G, Ives J, Bradbury-Jones C, Irvine F. What is 'moral distress'? A narrative synthesis of the literature. Nursing Ethics. 2017.

14. Fernandez-Parsons R, Rodriguez L, Goyal D. Moral Distress In Emergency Nurses. J Emerg Nurs. 2013;39:547-52.

15. Ohnishi K, Ohgushi $\mathrm{Y}$, Nakano M, Fujii H, Tanaka H, Kitaoka K, et al. Moral distress experienced by psychiatric nurses in japan. Nursing Ethics. 2010;17:726-40.

16. Razzaghi Kashani OM, Motevallian SA, Alizadegan S, Vaziri M Hossein Merghati Khoiee E, Goushegir SA, et al. Developing the Moral Distress Scale in the Population of Iranian Nurses. Iranian Journal of Psychiatry. 2008;55:4655

17. Nadere RNG, Hasemi E, Farahani AHM, Borhani F. Evaluation of nurses' moral distress in educational institutions -Darmany Shaheed Beheshti University in 1392. Medical Ethics. 2013;8:121-43.

18. Beikmoradi A, Rabiee S, Khatiban M, Cheraghi MA. Nurses distress in intensive care unit: a survey in teaching hospitals. J Med Ethics Hist Med. 2012;5:58-69.

19. Ebrahimi H, Kazemi A, Jafarabadi MA, Azarm A.Moral distress in nurses working in educational hospitals of Northwest Medical Universities of Iran. J Med Ethics Hist Med. 2013;6:80-8.

20. Masomi GJ, M. Siahtir M. et. al. The measures of Emergency Department in hospitals. Iranian Ministry of Health. Health Policy Breauea.

21. Noorossana R, Rezaeian S, Saghaei A, Karimzadeh Y, Moradi S. Measuring Service Quality in Emergency department Based on patient rights: a crosssectional study from Iran. Med Ethics. 2013:7:161-84.

22. Ameri M, Mirhashemi B, Hosseini SS.Moral distress and the contributing factors among nurses in different work environments. JNMS. 2015:2:44-9.

23. Borhani F, Abbaszadeh A, Mohamadi E, Ghasemi E, Hoseinabad-Farahani MJ. Moral sensitivity and moral distress in Iranian critical care nurses. Nursing Ethics. 2017;24:474-82.

24. Allari R, Abu-Moghli F. Moral Distress among Jordanian Critical Care Nurse and their Perception of Hospital Ethical Climate. Journal of Natural Sciences Research. 2013;3:144-53.

25. de Veer AJ, Francke AL, Struijs A, Willems DL. Determinants of Moral Distress In Daily Nursing Practice: A Cross Sectional Correlational Questionnaire Survey. Int J Nurs Stud. 2013;50:100-8. 